\title{
Bayesian Microscopy: Model Selection for Extracting Weak Nonlinearities from Scanning Probe Microscopy Data
}

Rama Vasudevan ${ }^{1}$, Kyle Kelley ${ }^{1}$, Eugene Eliseev ${ }^{2}$, Hiroshi Funakubo ${ }^{3}$, Stephen Jesse ${ }^{4}$, Anna Morozovska $^{2}$ and Sergei Kalinin ${ }^{1}$

${ }^{1}$ Oak Ridge National Laboratory, Oak Ridge, Tennessee, United States, ${ }^{2}$ National Academy of Sciences of Ukraine, Kyiv, Kyyiv, Ukraine, ${ }^{3}$ Tokyo Institute of Technology, Yokohama, Kanagawa, Japan, ${ }^{4}$ Oak Ridge National Laboratory, NA, Alabama, United States

Scanning probe microscopy (SPM) has emerged as perhaps the premiere tool for investigations of the functional properties of materials at the nanoscale, given the wide prevalence of commercially available SPMs, the ease of sample preparation, and the huge variety of modes that SPM offers including forcedistance curves that can enable probing of mechanical properties and e.g. protein unfolding, electrical modes including Kelvin probe force microscopy and piezoresponse force microscopy, and atomically resolved studies utilizing scanning tunnelling microscopy and related spectroscopies. For cantilever-based measurements, inferring material parameters often requires performing the measurement and then choosing a model for the geometry of the tip and modelling the cantilever dynamics. Both are difficult, but the latter is made more difficult by intrinsically high noise issues in SPM, along with the availability of several types of cantilever models to choose from. The question of which model is appropriate, and how much such models can be trusted, are rarely explored and rather are postulated apriori for each individual study, without further statistical testing or validation. Recently, advances in both computation and in the mathematics of Bayesian inference and Bayesian model selection have enabled the deployment of model selection algorithms that can be run on modern desktop PCs. Here, we explore the use of such model selection algorithms in the context of interpretation of piezoresponse force microscopy (PFM) data captured using the band-excitation (BE) approach [1]. Briefly, BE-PFM relies on capturing the deflection of the cantilever when in contact with a sample (generally piezo or ferroelectric) upon application of an $\mathrm{AC}$ voltage. The frequency of the excitation is varied to enable the response to be captured around the resonant frequency of the cantilever. Subsequent inverse Fourier transformation yields the signal in the frequency domain. We performed $\mathrm{BE}-\mathrm{PFM}$ imaging on $\mathrm{PbTiO}_{3}$ thin film sample. To analyse this data, we considered two models:

$$
\mu \frac{d^{2} u}{d t^{2}}+c \frac{d u}{d t}+k u+\lambda u^{3}=f \sin (\omega t)
$$

Equation (1) describes a Duffing oscillator where $\mathrm{u}$ is the displacement, $\mu$ is the effective mass, $\mathrm{c}$ is the damping coefficient, $\mathrm{k}$ is the linear stiffness and $\lambda$ is the nonlinear stiffness, when driven by a periodic driving force $\mathrm{f}$ with period $\omega$. When the nonlinear stiffness term is zero, equation (1) reduces to a simple harmonic oscillator (SHO) model and has been the preponderant model of choice in the past [1], as it has a simple analytical solution. On the other hand, for small $\lambda$ values it is possible to approximate the solution to (1) as 


$$
R \approx \sqrt{2} \tilde{f}\left(4(\widetilde{\omega}-1)^{2}+\tilde{c}^{2}+\sqrt{\left(4(\widetilde{\omega}-1)^{2}+\tilde{c}^{2}\right)^{2}+3(1-\widetilde{\omega}) \tilde{\lambda} \tilde{f}^{2}}\right)^{-\frac{1}{2}}
$$

For model selection, we first performed Bayesian inference on the PFM dataset, estimating the parameters of the SHO model and Duffing model by sampling using the Metropolis-Hastings method, all within the PyMC3 python package. Such inference provides us with the posteriors, i.e., the probability distributions of the parameters for both models. Using these posterior distributions enables calculation of the widely applicably information criterion [2] which assists in model selection when several candidate models are evaluated with the goal of selecting the one model which best describes the data. Our analysis revealed that in all cases, the Duffing model was preferred. Moreover, the estimated values of the (reduced) nonlinear term, plotted in Fig. 1(a) show that most of the domains do indeed display a small but nonzero level of nonlinearity. Moreover, the inferred variance (Fig. 1(b)) is higher in the domains than the domain walls. Interestingly, observation of the fits from the Duffing and SHO models to a single point spectrum shown in Fig. 1(c) indicate very little difference by eye; yet, the Duffing model is apparently preferred here by a very large ( >99\%) certainty according to the WAIC. This study shows the promise of modern Bayesian tools in extracting more subtle physical details from microscopy and can be expected to be equally applicable to electron microscopy [3].
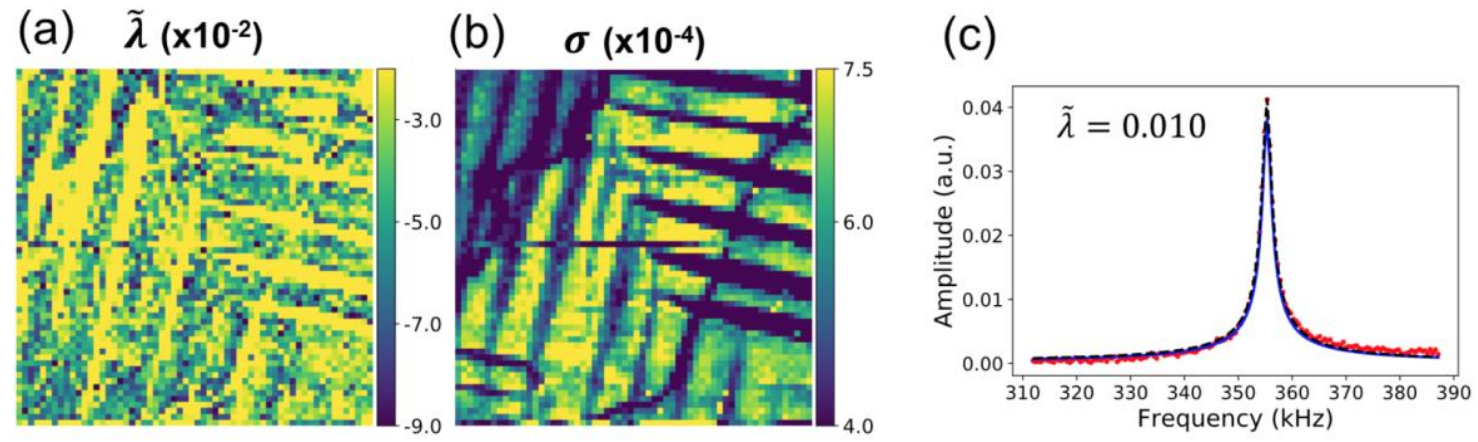

Figure 1. (a) Duffing oscillator model applied to piezoresponse force microscopy data, with estimates for the mean nonlinearity parameter (size of image: $400 \mathrm{x} 400 \mathrm{~nm}$ ) shown in (a), the estimated variance of the response (i.e., the noise level) in (b) and a single point spectra from a pixel in the same region shown in (c). the blue response is a SHO model and the black curve is from the Duffing model.

\section{References}

[1] S. Jesse, S. V. Kalinin, R. Proksch, A. P. Baddorf a B. J. Rodriguez, Nanotechnology 18 (2007), p. 435503

[2] S. Watanabe, J. Mach. Learn. Res. 14 (2013), p. 867

[3] This research was conducted at the Center for Nanophase Materials Sciences, which also provided support (R. K. V, S. J., S. V. K.) and is a US DOE Office of Science User Facility. 